\title{
Task Optimization for Spot-Welding by a Multiple Robot System Using Evolutionary Algorithm
}

\author{
Jahng Hyon Park, Jinhan Jeong \\ Hanyang University \\ 222 Wangsimni-ro, Seoul, Korea \\ jparkr@hanyang.ac.kr
}

\section{Extended Abstract}

Multiple spot-welding robots are used to improve productivity in automobile industry. A work cell considered in this paper consists of a number of robots working simultaneously for spot-welding in auto manufacturing process. Since the number of welding spots on a single car is very large, the sequence of tasks by the multiple-robots significantly affect the total tact time. The paths and sequences of the robots usually are generated using offline teaching method. This method highly depends not only on the intuition of human operator but also may cause inefficient productivity because of the nature of the cluttered environment with high degrees of freedom robots.

In this paper, the evolutionary algorithm is employed to allocate tasks to each robot and optimize the sequence of operations by the multiple robots. The evolutionary algorithm has been used for optimization problems with large and complex search spaces due to their ability of finding a near optimal solution [1]. In this paper, an optimization problem with multiple object function is formulated and a corresponding scheme is developed for coding with the evolutionary algorithm. Two types of chromosomes are employed for scheduling the spot-welding system using multi-objective genetic algorithm [2]. The first part of each chromosome represents a permutation of the $n$ welding spots and the second part of the chromosome represents the id of the assigned robots.

Creation of the offspring generations is performed by an operator which selects a chromosome by proportional roulette-wheel selection using the following fitness function.

$$
\mathrm{P}(\mathrm{i})=\mathrm{F}(\mathrm{i}) / \Sigma \mathrm{F}(\mathrm{j})
$$

where $\mathrm{P}(\mathrm{i})$ represents the value of fitness of an i-th individual.

The distance from the spot point to the end effector of the robot is used to select the mutation [3]. The mutation operator is used for the first part of the chromosome. The crossover operator is recombining the chromosome after reproduction of a selected identity. The role of this operator is joining parts of several individuals in order to produce new individuals for the next generation [4]. The one-point crossover is used [5] for operation on the second part of the chromosome.

The fitness function for one chromosome reflects how well the individual is adapted to the environment. The fitness function considered in this high-density robotic work cell is composed of three parts representing multiple objetives;

$$
\mathrm{F}=\alpha \mathrm{F}+\beta \mathrm{F}+\gamma \mathrm{F}
$$

The first objective function is included to reduce the total distance travelled by the robots. The second one is included to reflect the each welding time and the third one is calculating the distance between robots and welding points. Reducing the fitness function will lead to minimizing the total task time and thus improve productivity.

The proposed algorithm is applied to a system of 6 spot-welding robots with 338 welding spots. The simulation results show optimized load distribution and sequence of welding by each robot. This scheme of optimization is expected to be applied to other kinds of tasks other than spot welding by appropriately modifying the objective or fitness function and encoding the distribution and sequence into the chromosome. 


\section{Acknowledgement}

This work was supported by the research fund of Survivability Technology Defence Research Center of the Agency for Defence Development of Korea (No. UD150013ID).

\section{References}

[1] E. K. Xidias, P. Th. Zacharia, and N. A. Aspragathos, "Optimal task scheduling for a two-robot workcell," Journal of systems and control engineering, vol. 224, no. 7, pp. 845-855, 2010.

[2] S. Yuan, et al. "A new approach to solving the multiple traveling salesperson problem using genetic algorithms," European Journal of Operational Research, vol. 228, no. 1, pp. 72-82, 2013.

[3] K. Krishna and M. N. Murty, "Genetic K-Means Algorithm," in IEEE Transactions on system, Man, and Cybernetics Part B: Cybernetics, vol. 29, no. 3, 1999.

[4] P. T. Zacharia and N. A. Aspragathos, "Optimal robot task scheduling based on genetic algorithms," Robotics and Computer-Integrated Manufacturing, vol. 21, pp. 67-79, 2005.

[5] Z. Michalewitz, Genetic algorithms + data structures - evolution programs, 3rd Ed. Berlin: Springer, pp. 21, 219-20, 1996. 